\title{
Agentes infecciosos en pacientes pediátricos con diagnóstico de leucemia linfática aguda, aislados a partir de hemocultivos. Estudio preliminar
}

\author{
Yesica Llimpe, William Vicente, Helí Barrón
}

Instituto Nacional de Enfermedades Neoplásicas (INEN)

Objetivos: Identificar los agentes infecciosos más frecuentes aislados a partir de hemocultivos, en pacientes pediátricos con diagnóstico de leucemia linfática aguda (LLA).

Diseño: Descriptivo, observacional, retrospectivo.

Institución: Instituto Nacional de Enfermedades Neoplásicas (INEN).

Participantes: Pacientes menores de 15 años con diagnóstico de LLA y hemocultivo positivo.

Intervenciones: Revisión de historias clínicas de 89 pacientes menores de 15 años, admitidos en el INEN a partir de enero de 2008 a diciembre de 2009. Selección de casos con hemocultivo positivo.

Principales medidas de resultados: Número de episodios y agentes infecciosos.

Resultados: De 89 casos, 25 pacientes presentaron por lo menos un hemocultivo positivo. Se encontró 30 episodios infecciosos en los 25 pacientes, siendo los agentes infecciosos identificados: Staphylococcus sp. coagulasa negativa, Staphylococcus aureus, Klebsiella sp., Escherichia coli, Candida tropicalis, Pseudomonas aeruginosa, Staphylococcus intermedius, Streptococcus equimilis, Streptococcus acidominimus, Streptococcus pneumoniae, Streptococcus mitis, Enterobacter sp. y Salmonella sp.

Conclusiones: Los agentes infecciosos más frecuentes aislados a partir de hemocultivos de pacientes pediátricos con diagnóstico de LLA fueron Staphylococcus sp. coagulasa negativa, presentándose en $36,7 \%$ del total de episodios infecciosos.

Palabras clave: Agentes infecciosos, leucemia linfática aguda, pediatría.

\section{Estudio citogenético preliminar en las diferentes fases de la leucemia mieloide crónica, en pacientes del Instituto Nacional de Enfermedades Neoplásicas (INEN) 2006-2009}

José Ronceros, Yesica Llimpe, Abelardo Arias

Instituto Nacional de Enfermedades Neoplásicas (INEN).

Objetivos: Determinar las alteraciones cromosómicas en pacientes con leucemia mieloide crónica (LMC), durante su progresión.

Diseño: Estudio descriptivo retrospectivo longitudinal.

Institución: Instituto Nacional de Enfermedades Neoplásicas (INEN).

Participantes: Pacientes de ambos sexos con diagnostico de LMC.

Intervenciones: Se revisó las historias clínicas de 43 pacientes de ambos sexos admitidos en el INEN, cuyo diagnóstico fue LMC, y se determinó las frecuencias de alteraciones cromosómicas durante la progresión de la enfermedad en sus tres fases: fase crónica, fase acelerada y crisis blástica.

Principales medidas de resultados: Frecuencias de alteraciones cromosómicas.

Resultados: Los pacientes incluidos en el estudio presentaron una edad promedio de 38 años (rango de 15 a 79 años). La frecuencia de alteraciones cromosómicas observadas fue de 97,7\%. El cromosoma Philadelphia (Ph), producto de la translocación entre los cromosomas 9 y 22, se evidenció en $95 \%$ de casos totales, observándose en las tres fases. Otras alteraciones observadas fueron: +8 , del(22q), del(3p), $-11,-18$ y + mar.

Conclusiones: El 95\% de pacientes con diagnóstico de LMC presentó el cromosoma Ph, en algunos casos incluso se acompañó de alteraciones cromosómicas adicionales.

Palabras clave: Leucemia mieloide crónica, cromosoma Philadelphia. 